\title{
PALINDROMIC COMPLEXITY OF INFINITE WORDS ASSOCIATED WITH NON-SIMPLE PARRY NUMBERS
}

\author{
L'ubomíra Balková and ZuZana Masáková ${ }^{1}$
}

\begin{abstract}
We study the palindromic complexity of infinite words $u_{\beta}$, the fixed points of the substitution over a binary alphabet, $\varphi(0)=0^{a} 1$, $\varphi(1)=0^{b} 1$, with $a-1 \geq b \geq 1$, which are canonically associated with quadratic non-simple Parry numbers $\beta$.
\end{abstract}

1991 Mathematics Subject Classification. 68R15, 11A63 .

\section{IntRODUCTION}

Palindromes, words which stay the same when read backwards, is a popular linguistic game. The longest palindrome listed in Oxford Dictionary is "tattarrattat". This word arose in the fantasy of James Joyce and signified to tap on the door in his novel Ulysses. Not only words, but also palindromic numbers have been attracting attention for ages. Such numbers appear already in a sanskrit manuscript Ganitasarasâmgraha dated around 850 AD. However, palindromes occur in serious disciplines as well. For instance, nucleotides in most of human genomes form palindromic sequences.

In this paper, we focus on palindromes in infinite aperiodic words which can serve as models for one dimensional quasicrystals. Quasicrystals are materials with longrange orientational order demonstrated by sharp bright spots on their diffraction images, nevertheless, of non-crystallographic symmetry which testifies their aperiodicity. For their particular features, these materials have been in the center of interest of physicists, chemists, and mathematicians since their discovery in 1982 [19]. To understand physical properties of these materials, it is important to describe their combinatorial properties, such as factor complexity which corresponds to the number of local configurations of atoms, but also the palindromic structure which describes local symmetry of the material. Moreover, the palindromic structure of the infinite words turns out to be important for the description of the spectral properties of Schrödinger operators with potentials adapted to aperiodic structures [13].

Here, we concentrate on infinite words $u_{\beta}$ coding numbers with integer $\beta$-expansion. Such words are defined over a finite alphabet if the base $\beta$ of the numeration system is a Parry number, i.e., such that its Rényi expansion of unity $d_{\beta}(1)$ is eventually periodic. For the definition of $d_{\beta}(1)$ see Section 2. If $d_{\beta}(1)$ is finite, $\beta$ is called a simple Parry number. For such $\beta$, diverse combinatorial aspects of infinite words $u_{\beta}$ have been investigated. In [11], one describes the factor complexity of a

Keywords and phrases: Palindromes, beta-expansions, infinite words

${ }^{1}$ Doppler Institute for Mathematical Physics and Applied Mathematics \& Department of Mathematics, FNSPE, Czech Technical University, Trojanova 13, 12000 Praha 2, Czech Republic e-mail: 1.balkova@centrum.cz \& masakova@km1.fjfi.cvut.cz

(C) EDP Sciences 1999 
large subclass of these words. In [1], the palindromic complexity is given for the same subclass. In paper [5],the authors determine the number of return words. The balance properties of quadratic simple Parry numbers are studied in [21].

Much less is known about infinite words $u_{\beta}$ associated with non-simple Parry numbers. Some preliminary results about their factor complexity are given in [12] Otherwise, one finds concrete results only about infinite words $u_{\beta}$ where $\beta$ is a quadratic non-simple Parry number. In this case, $u_{\beta}$ is a fixed point of the substitution $\varphi(0)=0^{a} 1, \varphi(1)=0^{b} 1, a>b \geq 1$. The factor complexity of such infinite words has been determined in [11]. In [4], one studies their arithmetical and balance properties.

The aim of this paper is to determine the palindromic complexity of infinite words $u_{\beta}$ associated with non-simple Parry numbers. In fact, we explain that the only interesting case is again the case of quadratic non-simple Parry numbers, since the language of the infinite word $u_{\beta}$ for other non-simple Parry numbers $\beta$ contains only finitely many palindromes.

\section{Notations And Definitions}

\subsection{COMBINATORICS ON WORDS}

An alphabet $\mathcal{A}$ is a finite set of symbols called letters. A concatenation $w=$ $w_{1} w_{2} \cdots w_{n}$ of letters is called a word of length $|w|=n$. The set of all finite words, including the empty word $\varepsilon$, is denoted by $\mathcal{A}^{*}$, and has the structure of a free monoid with respect to the operation of concatenation. We deal also with rightsided infinite words, and, if need be, bidirectional infinite words, i.e., with sequences of letters $u=u_{0} u_{1} u_{2} \cdots$, or $u=\cdots u_{-1} u_{0} u_{1} u_{2} \cdots$, respectively. A finite word $w$ is called a factor of a word $u$ (finite or infinite) if there exist a finite word $w^{(1)}$ and a word $w^{(2)}$ (finite or infinite) such that $u=w^{(1)} w w^{(2)}$. The word $w$ is a prefix of $u$ if $w^{(1)}=\varepsilon$. Analogously, $w$ is a suffix of $u$ if $w^{(2)}=\varepsilon$. A concatenation of $k$ words $z$ will be denoted by $z^{k}$, a concatenation of infinitely many words $z$ by $z^{\omega}$. An infinite word $u$ is said to be eventually periodic if there exist words $w, z$ such that $u=w z^{\omega}$. Let $u=u_{1} u_{2} u_{3} \cdots, u_{i} \in \mathcal{A}$, then $u_{1}^{-1} u=u_{2} u_{3} \cdots$. The language of $u$, denoted by $\mathcal{L}(u)$, is the set of all factors of the word $u$. A letter $z \in \mathcal{A}$ is called a left extension of a factor $w$ of the word $u$ if $z w$ belongs to $\mathcal{L}(u)$. The factor $w$ is a left special factor of $u$ if $w$ has more than one left extensions. Similarly we define right special factors. The factor complexity (or simply complexity) of an infinite word $u$ is the function $\mathcal{C}: \mathbb{N} \rightarrow \mathbb{N}$ such that

$$
\mathcal{C}(n)=\text { the number of different factors of } u \text { of length } n \text {. }
$$

We will denote by $\bar{w}$ the reversal of $w$, i.e., if $w=w_{1} w_{2} \cdots w_{n}$, then $\bar{w}=$ $w_{n} \cdots w_{2} w_{1}$. A palindrome is a word $w$ which is equal to its reversal $\bar{w}$. The palindromic complexity of an infinite word $u$ is the function $\mathcal{P}: \mathbb{N} \rightarrow \mathbb{N}$ such that

$$
\mathcal{P}(n)=\text { the number of different palindromic factors of } u \text { of length } n \text {. }
$$

If for a palindrome $w \in \mathcal{L}(u)$ there exists $z \in \mathcal{A}$ such that $z w z$ is also a factor of $u$, then $z$ is a palindromic extension of $w$. The language of $u$ is closed under reversal if for every factor $w$ of $u$, also $\bar{w}$ is in $\mathcal{L}(u)$.

A substitution on $\mathcal{A}^{*}$ is a morphism $\varphi: \mathcal{A}^{*} \rightarrow \mathcal{A}^{*}$ such that there exists a letter $z \in \mathcal{A}$ and a non-empty word $w \in \mathcal{A}^{*}$ satisfying $\varphi(z)=z w$ and $\varphi(y) \neq \varepsilon$ for all $y \in \mathcal{A}$. Since any morphism satisfies $\varphi(v w)=\varphi(v) \varphi(w)$ for all $v, w \in \mathcal{A}^{*}$, it suffices to define the substitution over the alphabet $\mathcal{A}$. An infinite word $u$ is said to be 
a fixed point of the substitution $\varphi$ if it fulfills

$$
u=u_{1} u_{2} u_{3} \cdots=\varphi\left(u_{1}\right) \varphi\left(u_{2}\right) \varphi\left(u_{3}\right) \cdots=\varphi(u) .
$$

It is obvious that a substitution $\varphi$ has at least one fixed point, namely $\lim _{n \rightarrow \infty} \varphi^{n}(z)$.

A substitution $\varphi$ over the alphabet $\mathcal{A}$ is called primitive if there exists $k \in \mathbb{N}$ such that for any $z \in \mathcal{A}$ the word $\varphi^{k}(z)$ contains all the letters of $\mathcal{A}$. It has been proved [9] that if $u$ is a fixed point of a primitive substitution $\varphi$, then $u$ is uniformly recurrent, that is, for every $n \in \mathbb{N}$ there exists $R(n)>0$ such that any factor of $u$ of length $\geq R(n)$ contains all the factors of $u$ of length $n$.

\subsection{Beta-expansions, Beta-Integers, And RÉnyi EXPANSIOn of Unity}

Let $\beta>1$ be a real number. For any non-negative real number $x$ there exist a series of coefficients $\left(x_{i}\right)_{i=-\infty}^{k}$ such that $x_{i} \in \mathbb{N}$ satisfying

$$
0 \leq \sum_{i=N}^{k} x_{i} \beta^{i}<\beta^{N} \quad \text { for all } N \in \mathbb{Z}, N \leq k .
$$

The representation of $x$ in the form of the convergent series $x=\sum_{i=-\infty}^{k} x_{i} \beta^{i}$ is called the $\beta$-expansions of $x$. Note that the elements of the sequence $\left(x_{i}\right)_{i \leq k}$ can also be obtained using the mapping $T_{\beta}:[0,1] \mapsto[0,1)$ defined by $T_{\beta}(x):=\beta x-\lfloor\beta x\rfloor$, by the prescription $x_{i}=\left\lfloor\beta T_{\beta}^{k-i}\left(x / \beta^{k+1}\right)\right\rfloor$. This implies that the 'digits' $x_{i}$ of the $\beta$-expansion take values in the set $\{0,1, \ldots,\lceil\beta\rceil-1\}$.

Numbers $x \geq 0$ whose $\beta$-expansion is of the form $\sum_{i=0}^{k} x_{i} \beta^{i}$ are called nonnegative $\beta$-integers, their set is denoted $\mathbb{Z}_{\beta}^{+}$. The set of $\beta$-integers is $\mathbb{Z}_{\beta}=-\mathbb{Z}_{\beta}^{+} \cup \mathbb{Z}_{\beta}^{+}$. The properties of $\beta$-integers depend on the so-called Rényi expansion of unity in base $\beta$ defined as

$$
d_{\beta}(1)=t_{1} t_{2} t_{3} \cdots, \quad \text { where } \quad t_{i}:=\left\lfloor\beta T_{\beta}^{i-1}(1)\right\rfloor .
$$

Note that $t_{1}=\lfloor\beta\rfloor \geq 1$. It is interesting to mention that not every sequence of nonnegative integers is equal to $d_{\beta}(1)$ for some $\beta$. Parry studied this problem in his paper [16] and showed that a sequence $\left(t_{i}\right)_{i \geq 1}, t_{i} \in \mathbb{N}$, is the Rényi expansion of unity for some number $\beta$ if and only if the sequence satisfies

$$
t_{j} t_{j+1} t_{j+2} \ldots \prec t_{1} t_{2} t_{3} \ldots \quad \text { for every } j>1 \text {, }
$$

where $\prec$ stands for strictly lexicographically smaller.

\subsection{INFINITE WORDS ASSOCIATED WITH BETA-INTEGERS}

If $\beta$ is an integer, then $\mathbb{Z}_{\beta}=\mathbb{Z}$. For $\beta \notin \mathbb{N}$, Thurston [20] has shown that the distances between consecutive $\beta$-integers take values in the set $\left\{\Delta_{k} \mid k \in \mathbb{N}\right\}$, where

$$
\Delta_{k}:=\sum_{i=1}^{\infty} \frac{t_{i+k}}{\beta^{i}} \text { for } k \in \mathbb{N}_{0}
$$

It is evident that the set $\left\{\Delta_{k} \mid k \in \mathbb{N}_{0}\right\}$ is finite if and only if $d_{\beta}(1)$ is eventually periodic. Numbers $\beta$ with eventually periodic Rényi expansion of unity are called Parry numbers. Coding the distances $\Delta_{i}$ by letters, the set $\mathbb{Z}_{\beta}^{+}$of non-negative integers can be naturally represented by an infinite word over a finite alphabet; such a right-sided infinite word is usually denoted by $u_{\beta}$. 
If the Rényi expansion of unity in base $\beta$ is finite (i.e., $d_{\beta}(1)$ ends in infinitely many 0's), $\beta$ is said to be a simple Parry number. In this paper, we focus on nonsimple Parry numbers $\beta$, i.e., numbers having eventually periodic, but infinite Rényi expansion of unity. Let $\beta$ be a non-simple Parry number and let $m, p$ be minimal such that

$$
d_{\beta}(1)=t_{1} \ldots t_{m}\left(t_{m+1} \ldots t_{m+p-1}\right)^{\omega}
$$

is the Rényi expansion of unity in base $\beta$. In this case, there are $m+p-1$ different distances between neighboring $\beta$-integers. If we assign the letter $i$ to the distance $\Delta_{i}$, then $\mathbb{Z}_{\beta}^{+}$is represented by an infinite word $u_{\beta}$ over the alphabet $i \in\{0, \ldots, m+p-1\}$. It has been proved [10] that the infinite word $u_{\beta}$ is the unique fixed point of the following primitive substitution:

$$
\begin{aligned}
\varphi(j) & =0^{t_{j}}(j+1) \quad \text { for } j \in\{0,1, \ldots, m+p-2\}, \\
\varphi(m+p-1) & =0^{t_{m+p}} m .
\end{aligned}
$$

\section{LANGuages Closed under REVERSAl FOR PARRY Numbers}

The existence of palindromes in an infinite word is strongly related with invariance under reversal of the corresponding language. This property singles out among all the words $u_{\beta}$ with non-simple Parry number $\beta$ those, for which $\beta$ is a quadratic number.

We remind two results; at first, a proposition, proved in [1], which explains how the number of palindromes is connected with the invariance of the language $\mathcal{L}\left(u_{\beta}\right)$ under reversal.

Proposition 3.1. Let $u$ be an infinite uniformly recurrent word. If for every $n \in \mathbb{N}$ there exists a palindrome in $\mathcal{L}(u)$ of length greater than $n$, then $\mathcal{L}(u)$ is closed under reversal.

It follows immediately that if there exists a factor $w \in \mathcal{L}(u)$ such that its reversal $\bar{w} \notin \mathcal{L}(u)$, then there is only a finite number of palindromes in $\mathcal{L}(u)$. The following proposition taken from [6] determines the non-simple Parry numbers $\beta$, for which the language $\mathcal{L}\left(u_{\beta}\right)$ is closed under reversal.

Proposition 3.2. Let $\beta$ be a non-simple Parry number with the Rényi expansion of unity $d_{\beta}(1)=t_{1} \ldots t_{m}\left(t_{m+1} \ldots t_{m+p-1}\right)^{\omega}$, where $m$, $p$ are minimal, and let $u_{\beta}$ be the fixed point of the substitution (4). The language $\mathcal{L}\left(u_{\beta}\right)$ is closed under reversal if and only if $m=p=1$.

The condition $m=p=1$ in the above proposition implies that the Rényi expansion of unity of the number $\beta$ is of the form

$$
d_{\beta}(1)=a b^{\omega}, \quad \text { where } a-1 \geq b \geq 1 .
$$

Consequently, $\beta$ is the larger root of the polynomial $x^{2}-(a+1) x+a-b$, the substitution $\varphi$ is defined over the alphabet $\{0,1\}$ by

$$
\varphi(0)=0^{a} 1, \quad \varphi(1)=0^{b} 1,
$$

and its fixed point $u_{\beta}=\lim _{n \rightarrow \infty} \varphi^{n}(0)$ is a right-sided infinite word of the form

$$
u_{\beta}=\underbrace{0^{a} 1 \cdots 0^{a} 1}_{a \text { times }} 0^{b} 1 \underbrace{0^{a} 1 \cdots 0^{a} 1}_{a \text { times }} 0^{b} 1 \cdots
$$

As a result of Propositions 3.1 and 3.2 if $\beta$ is a non-simple Parry number which is not quadratic, then the language of the infinite word $u_{\beta}$ contains only a finite 
number of palindromes. Therefore in the study of palindromic complexity of infinite words $u_{\beta}$, we will be particularly interested in quadratic non-simple Parry numbers with $d_{\beta}(1)$ of the form (5).

The case of $b=a-1$ has been already studied and the palindromic complexity of the corresponding infinite word $u_{\beta}$ is known. If $b=a-1$, then $\beta$ is the larger root of the polynomial $x^{2}-(a+1) x+1$, i.e., $\beta$ is a quadratic Pisot unit. In [7], it has been shown that the complexity of $u_{\beta}$ in this case is $\mathcal{C}(n)=n+1$ for $n \in \mathbb{N}$, i.e., $\mathcal{C}(n)$ is the smallest possible for aperiodic words. Infinite words having the smallest possible complexity are called Sturmian words. It has been shown in [8] that each palindrome $p$ of a Sturmian word $u$ has exactly one palindromic extension, i.e., there exists exactly one letter $z \in\{0,1\}$, such that $z p z \in \mathcal{L}(u)$. Consequently, there are exactly 2 palindromes of any odd length and one palindrome of any even length, i.e.,

$$
P(2 n)=P(0)=1 \quad \text { and } \quad P(2 n+1)=P(1)=2 \quad \text { for all } n \in \mathbb{N} .
$$

From now on, we will limit our considerations to quadratic non-simple and nonSturmian Parry numbers with the Rényi expansion of unity equal to $d_{\beta}(1)=a b^{\omega}$, where $a-1>b \geq 1$.

\section{FACtor COMPlexity of $u_{\beta}$}

Let us recall some results from [3,12] concerning the factor complexity of the infinite words $u_{\beta}$ for a quadratic non-simple Parry number $\beta$. It turns out that the notions, tools, and ideas developed for determining the factor complexity of $u_{\beta}$ will be useful also in determining its palindromic complexity.

Recall that in order to describe the factor complexity of an infinite word, it suffices to describe its left special factors. For the case of an infinite word over a binary alphabet, the reason is particularly simple. Denoting by $M_{n}$ the set of all left special factors of length $n$, it is obvious that the first difference of complexity satisfies

$$
\Delta C(n)=C(n+1)-C(n)=\# M_{n} .
$$

The first observation about special factors is presented by the following lemma.

Lemma 4.1. Let $10^{r} 1$ be a factor of $u_{\beta}$, then either $r=a$ or $r=b$. Let $v$ be a left (right) special factor of $u_{\beta}$ containing the letter 1 , then $v$ has the prefix $0^{b} 1$ (the suffix $\left.10^{b}\right)$.

Proof. The first statement follows immediately from the form of substitution (6). See (7) to understand that there are no other blocks of the form $10^{r} 1$. Consequently, a left special factor must have the prefix $0^{b} 1$, otherwise, it would have a unique left extension.

Taking a factor $w$ of $u_{\beta}$, by Lemma 4.1, the word $0^{b} 1 \varphi(w) 0^{b}$ is also a factor of $u_{\beta}$. On the other hand, every factor of $u_{\beta}$ with the prefix $0^{b} 1$ and suffix $10^{b}$ can be written as $0^{b} 1 \varphi(w) 0^{b}$ for some factor $w$ of $u_{\beta}$. Thus, defining a mapping $T:\{0,1\}^{*} \rightarrow\{0,1\}^{*}$ which will be very useful throughout the paper, we have

$$
w \in \mathcal{L}\left(u_{\beta}\right) \quad \Longleftrightarrow \quad T(w):=0^{b} 1 \varphi(w) 0^{b} \in \mathcal{L}\left(u_{\beta}\right) .
$$

For the description of the left special factors of $u_{\beta}$, one introduces the following notions.

Definition 4.2. Let $u_{\beta}$ be the infinite word associated with $d_{\beta}(1)=a b^{\omega}, a-1>$ $b \geq 1$.

- An infinite word $v$ is called an infinite left special branch of $u_{\beta}$ if each prefix of $v$ is a left special factor of $u_{\beta}$. 
- A left special factor $w \in \mathcal{L}\left(u_{\beta}\right)$ is called maximal if neither $w 0$ nor $w 1$ are left special.

- A factor $w$ of $u_{\beta}$ is called total bispecial if both $w 0$ and $w 1$ are left special factors of $u_{\beta}$.

The following facts, proved in [12], represent the consecutive steps needed for the determination of the factor complexity of $u_{\beta}$ :

- Every left special factor is a prefix of a maximal left special factor or of an infinite left special branch. A total bispecial factor is a common prefix of an infinite left special branch and a maximal left special factor.

- The only maximal left special factor which does not contain the letter 1 has the form $0^{a-1}$ and the only total bispecial factor containing no letter 1 is $0^{b}$.

- Every maximal left special (total bispecial) factor of $u_{\beta}$ that contains at least one letter 1 is also right special, and therefore by Lemma 4.1. can be written as $0^{b} 1 \varphi(w) 0^{b}$ for some maximal left special (total bispecial) factor $w$.

- If $w \in \mathcal{L}\left(u_{\beta}\right)$, then $w$ is a left special factor if and only if $T(w)$ is a left special factor. Moreover, $w$ is maximal if and only if $T(w)$ is maximal and $w$ is total bispecial if and only if $T(w)$ is total bispecial.

- From the previous, all maximal left special factors have the form:

$$
\begin{aligned}
U^{(1)} & =0^{a-1}, \\
U^{(n)} & =T\left(U^{(n-1)}\right) \quad \text { for } n \geq 2 .
\end{aligned}
$$

- All total bispecial factors have the form:

$$
\begin{aligned}
V^{(1)} & =0^{b}, \\
V^{(n)} & =T\left(V^{(n-1)}\right) \quad \text { for } n \geq 2 .
\end{aligned}
$$

- $V^{(n-1)}$ is a prefix of $V^{(n)}, V^{(n)}$ is a prefix of $U^{(n)}$, and

$$
\left|V^{(n)}\right|<\left|U^{(n)}\right|<\left|V^{(n+1)}\right| \quad \text { for all } n \in \mathbb{N} .
$$

- There exists a unique infinite left special branch of $u_{\beta}$, namely the infinite word $\lim _{n \rightarrow \infty} V^{(n)}$.

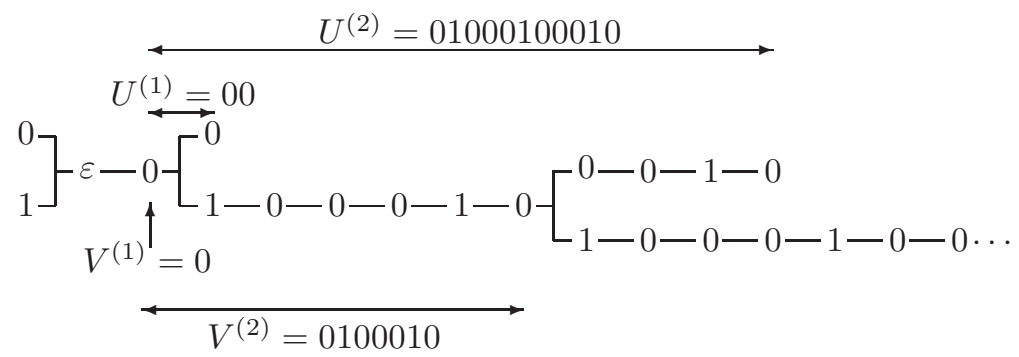

FigURE 1. Illustration of the tree of left special factors for $u_{\beta}$ being the fixed point of the substitution $\varphi(0)=0001, \varphi(1)=01$. We can see total bispecial factors $V^{(k)}$ and maximal left special factors $U^{(k)}$ for $k=1,2$. 
If we sum up the previous results, we notice that there is one left special factor of length $n$ being the prefix of the infinite left special branch for any $n \in \mathbb{N}$. Since $\left|V^{(k)}\right|<\left|U^{(k)}\right|<\left|V^{(k+1)}\right|$ and $V^{(k)}$ is a prefix of $U^{(k)}$, there exists a left special factor of length $n$ being prefix of $U^{(k)}$ and not prefix of $V^{(k)}$ if $\left|V^{(k)}\right|<n \leq\left|U^{(k)}\right|$. Thus, we obtain the following theorem.

Theorem 4.3. Let $u_{\beta}$ be the fixed point of the substitution $\varphi(0)=0^{a} 1, \varphi(1)=0^{b} 1$ for $a-1>b \geq 1$. Then for all $n \in \mathbb{N}$,

$$
\Delta C(n)= \begin{cases}2 & \text { if }\left|V^{(k)}\right|<n \leq\left|U^{(k)}\right| \text { for some } k \in \mathbb{N}, \\ 1 & \text { otherwise. }\end{cases}
$$

\section{Palindromic Complexity of $u_{\beta}$}

In this section, we reach the main goal of this paper which is to determine the palindromic complexity $\mathcal{P}(n)$ of infinite words associated with quadratic non-simple Parry numbers $\beta$ with the Rényi expansion $d_{\beta}(1)=a b^{\omega}$. As we have mentioned, the case $b=a-1$ has been already solved, the infinite word $u_{\beta}$ being Sturmian under this assumption. Thus we limit our considerations to the case of $a-1>b$. We proceed in more steps: First, we investigate palindromic extensions of palindromic factors of $u_{\beta}$. Second, we study centers of palindromes, and, finally, we focus on infinite palindromic branches. This allows us to conclude by determination of the palindromic complexity.

\subsection{Palindromic extensions of Palindromic FaCtors of $u_{\beta}$}

Since the word $u_{\beta}$ is defined over a binary alphabet, a palindrome in $u_{\beta}$ can have two, one, or no palindromic extensions. It turns out that most usually, palindromes in $u_{\beta}$ have exactly one palindromic extension. In this section, we determine the exceptional palindromes, i.e., those that do not have any palindromic extensions (called maximal palindromes), and those that have two palindromic extensions.

Definition 5.1. Let $p$ and $q$ be palindromes in $\mathcal{L}\left(u_{\beta}\right)$.

- The palindrome $p$ is a central factor of the palindrome $q$ if there exists a finite word $w \in \mathcal{L}\left(u_{\beta}\right)$ such that $q=w p \bar{w}$.

- The palindrome $p$ is called maximal if neither $0 p 0$ nor $1 p 1$ belong to $\mathcal{L}\left(u_{\beta}\right)$, i.e., if the only palindrome having $p$ as its central factor is $p$ itself.

Every palindrome is either a central factor of a maximal palindrome or a central factor of a sequence of palindromes with increasing length. In the latter case, we will speak about infinite palindromic branches. The exact definition and description of infinite palindromic branches in $u_{\beta}$ follows in Section 5.3

Let us now determine all maximal palindromes in $u_{\beta}$. First, realize a simple consequence of Lemma 4.1 .

Lemma 5.2. Let $p$ be a palindrome of $u_{\beta}$ containing at least one letter 1. Then $p$ is a central factor of a palindrome with prefix $0^{b} 1$ (and, equivalently, suffix $10^{b}$ ).

Consequently, a palindrome which has not the prefix $0^{b} 1$ is either not maximal, or does not contain the letter 1 . Such palindrome must be of the form $0^{r}$ for some $1 \leq r \leq a$, and it can be easily shown that the only maximal palindrome of such form is $0^{a-1}$.

In order to determine all maximal palindromes of $u_{\beta}$, let us now study the behaviour of palindromes with respect to the substitution $\varphi$. Since every maximal palindrome $p$ containing the letter 1 has the prefix $0^{b} 1$ and the suffix $10^{b}$, one can always write it as $0^{b} 1 \varphi(q) 0^{b}$ for some factor $q \in \mathcal{L}\left(u_{\beta}\right)$. The following lemma shows 
that similarly as in the case of left special factors studied for determining the factor complexity, we can use the substitution $\varphi$ to reduce all maximal palindromes containing at least one letter 1 to the maximal palindrome $0^{a-1}$.

Lemma 5.3. Let $p \in \mathcal{L}\left(u_{\beta}\right)$. Then $p$ is a palindrome in $u_{\beta}$ if and only if $T(p)=$ $0^{b} 1 \varphi(p) 0^{b}$ is a palindrome in $u_{\beta}$. Moreover,

$$
\left\{z \in\{0,1\} \mid z p z \in \mathcal{L}\left(u_{\beta}\right)\right\}=\left\{z \in\{0,1\} \mid z T(p) z \in \mathcal{L}\left(u_{\beta}\right)\right\} .
$$

Proof. Let us investigate under which condition $0^{b} 1 \varphi(p) 0^{b}$ is a palindrome in $u_{\beta}$. We will study for which $p$ it holds that

$$
0^{b} \overline{\varphi(p)} 10^{b}=0^{b} 1 \varphi(p) 0^{b} .
$$

Note that we can write $\overline{\varphi(0)}=10^{a}=1 \varphi(0) 1^{-1}$ and $\overline{\varphi(1)}=10^{b}=1 \varphi(1) 1^{-1}$. Then denoting $p=p_{1} \ldots p_{k}$, we have

$$
\overline{\varphi(p)} 1=\overline{\varphi\left(p_{1}\right) \ldots \varphi\left(p_{k}\right)} 1=\overline{\varphi\left(p_{k}\right)} \ldots \overline{\varphi\left(p_{1}\right)} 1=1 \varphi(\bar{p}) .
$$

As $\varphi(p)=\varphi(\bar{p})$ if and only if $p=\bar{p}$, the first statement of the lemma is proved.

Let us now verify $\left\{z \in\{0,1\} \mid z p z \in \mathcal{L}\left(u_{\beta}\right)\right\}=\left\{z \in\{0,1\} \mid z T(p) z \in \mathcal{L}\left(u_{\beta}\right)\right\}$.

$\subset$ Let $0 p 0 \in \mathcal{L}\left(u_{\beta}\right)$. Then $T(0 p 0)=0^{b} 1 \varphi(0) \varphi(p) \varphi(0) 0^{b} \in \mathcal{L}\left(u_{\beta}\right)$. Since $\varphi(0)$ has the suffix $00^{b} 1$ and the prefix $0^{b} 0$, we have $0 T(p) 0 \in \mathcal{L}\left(u_{\beta}\right)$.

Let now $1 p 1 \in \mathcal{L}\left(u_{\beta}\right)$. Then $T(1 p 1)=0^{b} 1 \varphi(1) \varphi(p) \varphi(1) 0^{b} \in \mathcal{L}\left(u_{\beta}\right)$ and from $\varphi(1)=0^{b} 1$, we deduce that $1 T(p) 1 \in \mathcal{L}\left(u_{\beta}\right)$.

$\supset$ Let $0 T(p) 0 \in \mathcal{L}\left(u_{\beta}\right)$. Due to Lemma 4.1 $0 T(p) 0=0^{b+1} 1 \varphi(p) 0^{b+1}$ can be uniquely extended on both sides to the factor

$$
0^{b} 10^{a} 1 \varphi(p) 0^{a} 10^{b}=0^{b} 1 \varphi(0 p 0) 0^{b}=T(0 p 0) \in \mathcal{L}\left(u_{\beta}\right),
$$

which implies $0 p 0 \in \mathcal{L}\left(u_{\beta}\right)$.

Similarly, let $1 T(p) 1 \in \mathcal{L}\left(u_{\beta}\right)$. Then $1 T(p) 1=10^{b} 1 \varphi(p) 0^{b} 1$ is a central factor of $0^{b} 10^{b} 1 \varphi(p) 0^{b} 10^{b}=0^{b} 1 \varphi(1 p 1) 0^{b}=T(1 p 1) \in \mathcal{L}\left(u_{\beta}\right)$, which implies $1 p 1 \in \mathcal{L}\left(u_{\beta}\right)$.

Using Lemma 5.3, we can see that the maximal left special factors $U^{(n)}$, defined by (10), are at the same time maximal palindromes.

Proposition 5.4. Let $p$ be a palindrome in $u_{\beta}$. Then $p$ is a maximal palindrome if and only if $p=U^{(n)}$ for a positive integer $n$.

Proof. From their definition by (10) and from Lemma 5.3, every $U^{(n)}$ is a maximal palindrome. We show the opposite implication by induction on the number of letters 1 contained in $p$. If $p$ does not contain 1 and $p$ is maximal, then $p$ is equal to $U^{(1)}=0^{a-1}$. There is no maximal palindrome containing only one letter 1 . Let $p$ contain at least two letters 1 . Then by Lemma $5.2 p$ is of the form $p=0^{b} 1 \varphi(q) 0^{b}$. Using Lemma 5.3. we know that $q$ is a maximal palindrome and $q$ contains a smaller number of letters 1 than $p$. By induction hypothesis, $q=U^{(k)}$ for some $k \in \mathbb{N}$ and $p=0^{b} 1 \varphi\left(U^{(k)}\right) 0^{b}=U^{(k+1)}$.

Let us now determine the palindromes which have two palindromic extensions. Such a palindrome is both a left special and a right special factor of $u_{\beta}$. Therefore by Lemma 4.1. it must have the prefix $0^{b} 1$ and the suffix $10^{b}$, whenever it contains at least one letter 1 . Thus again, we can write such a palindrome $p$ as $p=0^{b} 1 \varphi(q) 0^{b}$ 
for some $q \in \mathcal{L}\left(u_{\beta}\right)$. Now it suffices to realize that the only palindrome of the form $0^{r}$ with two palindromic extensions, is $V^{(1)}=0^{b}$. Using Lemma 5.3 we deduce that the total bispecial factors $V^{(n)}$ defined by (11) have two palindromic extensions.

Proposition 5.5. Let $p$ be a palindrome in $u_{\beta}$. Then $p$ has two palindromic extensions if and only if $p=V^{(n)}$ for a positive integer $n$.

Proof. From Lemma 5.3, it is clear that palindromes $V^{(n)}$ have two palindromic extensions. It suffices to show that any palindrome with two palindromic extensions is equal to some $V^{(n)}$. Let $p$ be a palindrome such that $0 p 0,1 p 1 \in \operatorname{Pal}\left(u_{\beta}\right)$, then $p$ is a left special factor and it is not maximal. Without loss of generality, we can suppose that $p 0$ is a left special factor, i.e., $0 p 0,1 p 0 \in \mathcal{L}\left(u_{\beta}\right)$. Using the fact that $\mathcal{L}\left(u_{\beta}\right)$ is closed under reversal, we have $0 p 1,1 p 1 \in \mathcal{L}\left(u_{\beta}\right)$. It means that also $p 1$ is a left special factor. Consequently, $p$ is a total bispecial factor, i.e., $p=V^{(k)}$ for some $k \in \mathbb{N}$.

Let us summarize the results of this section by classifying palindromes in $u_{\beta}$ according to their number of palindromic extensions. This a crucial step for the determining of palindromic complexity of $u_{\beta}$. It is interesting to notice that sequences $U^{(n)}$ of maximal left special factors and $V^{(n)}$ of total bispecial factors play an mportant role not only in computing the factor complexity, but also in determining the palindromic complexity. For illustration, consider the example from Figure 1 in Section 4 and check that the total bispecial factors and maximal left special factors illustrated there are indeed palindromes.

Proposition 5.6. Let $p$ be a palindrome in $u_{\beta}$. Then

(1) $p$ is a maximal palindrome $\Leftrightarrow p=U^{(n)}$ for a positive integer $n$.

(2) $p$ has two palindromic extensions $\Leftrightarrow p=V^{(n)}$ for a positive integer $n$.

(3) $p$ has one palindromic extension $\Leftrightarrow p \neq U^{(n)} \wedge p \neq V^{(n)}$ for all $n \in \mathbb{N}$.

Using the inequality $\left|V^{(n)}\right|<\left|U^{(n)}\right|<\left|V^{(n+1)}\right|$ for all $n \in \mathbb{N}$, we obtain the following corollary of Proposition 5.6 .

Corollary 5.7. Let $u_{\beta}$ be the fixed point of the substitution $\varphi(0)=0^{a} 1, \varphi(1)=$ $0^{b} 1, a-1>b \geq 1$. Then

$$
\mathcal{P}(n+2)-\mathcal{P}(n)=\left\{\begin{aligned}
1 & \text { if } n=\left|V^{(k)}\right| \text { for a positive integer } k \\
-1 & \text { if } n=\left|U^{(k)}\right| \text { for a positive integer } k \\
0 & \text { otherwise }
\end{aligned}\right.
$$

Combining with Theorem 4.3 we can derive a simple connection of palindromic complexity with the second difference of factor complexity:

$$
\Delta^{2} \mathcal{C}(n)=\Delta \mathcal{C}(n+1)-\Delta \mathcal{C}(n)=\mathcal{P}(n+2)-\mathcal{P}(n) .
$$

This relation is essential to show the following corollary.

Corollary 5.8. Let $u_{\beta}$ be the fixed point of the substitution $\varphi(0)=0^{a} 1, \varphi(1)=$ $0^{b} 1, a-1>b \geq 1$. Then

$$
\mathcal{P}(n+1)+\mathcal{P}(n)=\Delta \mathcal{C}(n)+2 \quad \text { for all } n \in \mathbb{N}
$$

Proof. We have $\mathcal{P}(n+1)+\mathcal{P}(n)=\mathcal{P}(0)+\mathcal{P}(1)+\sum_{i=1}^{n}(\mathcal{P}(i+1)-\mathcal{P}(i-1))=$ $=1+2+\sum_{i=1}^{n}(\Delta \mathcal{C}(i)-\Delta \mathcal{C}(i-1))=3+\Delta \mathcal{C}(n)-\mathcal{C}(1)+\mathcal{C}(0)=\Delta \mathcal{C}(n)+2$. 
Let us mention that for uniformly recurrent infinite words in general, one has $\mathcal{P}(n+1)+\mathcal{P}(n) \leq \Delta \mathcal{C}(n)+2$ for all $n \in \mathbb{N}$. This is shown in [2] together with several examples of infinite words where the equality is reached. By Corollary 5.8, we have found yet another class of infinite words with this property.

A direct consequence of the above corollary is the fact that the palindromic complexity of $u_{\beta}$ is bounded since $P(n+1)+P(n) \leq 4$.

\subsection{Centers of PALindromes}

We have seen that the set of palindromes of $u_{\beta}$ is closed under the mapping $p \mapsto$ $T(p)=0^{b} 1 \varphi(p) 0^{b}$. Let us study how $T$ acts on the central factors of palindromes.

Definition 5.9. Let $p \in \mathcal{L}\left(u_{\beta}\right)$ be a palindrome of odd length. The center of $p$ is a letter $z \in\{0,1\}$ such that $p=w z \bar{w}$, where $w \in \mathcal{L}\left(u_{\beta}\right)$. The center of a palindrome of even length is the empty word.

Lemma 5.10. Let $p, q$ be palindromes in $u_{\beta}$. If $q$ is a central factor of $p$, then $T(q)$ is a central factor of $T(p)$.

Proof. Let $p=w 0 q 0 \bar{w}$ for a $w \in \mathcal{L}\left(u_{\beta}\right)$. Then $T(p)=0^{b} 1 \varphi(w) 0^{a} 1 \varphi(q) 0^{a} 1 \varphi(\bar{w}) 0^{b}$. According to Lemma 5.3. $T(p)$ is a palindrome, and clearly, $T(q)=0^{b} 1 \varphi(q) 0^{b}$ is its central factor. The proof is similar for $p=w 1 q 1 \bar{w}$.

Now, using Lemma 5.10, we can describe how the center of the palindrome $T(p)$ depends on the center of the palindrome $p$.

Lemma 5.11. Let $p$ be a palindrome in $u_{\beta}$.

(i) If $p$ has the center $\varepsilon$, then $T(p)$ has the center 1 .

(ii) If $p$ has the center 0 , then $T(p)$ has the center $\begin{cases}0 & \text { for a odd, } \\ \varepsilon & \text { for a even. }\end{cases}$

(iii) If $p$ has the center 1 , then $T(p)$ has the center $\begin{cases}0 & \text { for } b \text { odd, } \\ \varepsilon & \text { for } b \text { even. }\end{cases}$

Proof. Let us verify for example the statement (ii). Using Lemma 5.10 it is evident that if $p$ has the center 0 , then $T(p)$ has the central factor $T(0)=0^{b} 10^{a} 10^{b}$. Consequently, the center of $T(p)$ is either 0 if $a$ is odd, or $\varepsilon$ if $a$ is even. Statements (i) and (iii) can be proved analogously.

Lemmas 5.10 and 5.11 allow us to describe the centers of palindromes with two palindromic extensions $V^{(n)}$ and the centers of maximal palindromes $U^{(n)}$.

Proposition 5.12. The centers and central factors of palindromes $V^{(n)}$ with two palindromic extensions depend on the values of parameters $a, b$.

(i) Let $b$ be even. Then for all $n \in \mathbb{N}, V^{(n)}$ is a central factor of $V^{(n+2)}$. Moreover, $V^{(2 n)}$ has the center 1 and $V^{(2 n-1)}$ has the center $\varepsilon$.

(ii) Let $b$ be odd and a even. Then for all $n \in \mathbb{N}, V^{(n)}$ is a central factor of $V^{(n+3)}$. Moreover, $V^{(3 n)}$ has the center $1, V^{(3 n-1)}$ has the center $\varepsilon$, and $V^{(3 n-2)}$ has the center 0 .

(iii) Let both $b$ and $a$ be odd. Then for all $n \in \mathbb{N}, V^{(n)}$ is a central factor of $V^{(n+1)}$ and has the center 0 .

Proof. In order to show the statement (i), it suffices to verify that $V^{(1)}$ is a central factor of $V^{(3)}$ and that $V^{(1)}$ has the center $\varepsilon$ and $V^{(2)}$ has the center 1 . The statement (i) then follows by induction on $n \in \mathbb{N}$. Since $b$ is even, $V^{(1)}=0^{b}$ has the center $\varepsilon$. By Lemma 5.11, $V^{(2)}$ has the center 1. Applying Lemma 5.10, one can see that $V^{(3)}$ has the central factor $T(1)=0^{b} 10^{b} 10^{b}$, i.e., it has also $V^{(1)}$ as its central factor.

Proofs of statements (ii) and (iii) are analogous. 
Proposition 5.13. The centers and central factors of maximal palindromes $U^{(n)}$ depend on the values of $a$ and $b$. Let $n \in \mathbb{N}$, we have the following cases:

(i) Let $b$ be even and $a$ odd. Then for all $n \in \mathbb{N}, V^{(n)}$ is a central factor of $U^{(n)}$. Moreover, $U^{(2 n-1)}$ has the center $\varepsilon$ and $U^{(2 n)}$ has the center 1 .

(ii) Let both $b$ and $a$ be even. Then $U^{(1)}=0^{a-1}$ is the only maximal palindrome with the center 0 . For all $n \in \mathbb{N}, V^{(n)}$ is a central factor of $U^{(n+1)}$. Moreover, $U^{(2 n)}$ has the center $\varepsilon$ and $U^{(2 n+1)}$ has the center 1.

(iii) Let $b$ be odd and a even. Then for all $n \in \mathbb{N}, V^{(n)}$ is a central factor of $U^{(n)}$. Moreover, $U^{(3 n-2)}$ has the center $0, U^{(3 n-1)}$ has the center $\varepsilon$, and $U^{(3 n)}$ has the center 1 .

(iv) Let both $b$ and $a$ be odd. The only maximal palindrome with the center $\varepsilon$ is $U^{(1)}=0^{a-1}$. The only maximal palindrome having the center 1 is $U^{(2)}$. For $n \geq 3, U^{(n)}$ has the center 0 and the central factor $V^{(n-2)}$.

Proof. Let us show for example the statement (ii). Since $a$ is even, $U^{(1)}=0^{a-1}$ has the center 0, Lemma 5.11 implies that $U^{(2)}$ has the center $\varepsilon$ and the central factor $T(0)=0^{b} 10^{a} 10^{b}$. Consequently, $V^{(1)}=0^{b}$ is also a central factor of $U^{(2)}, b$ being even. Applying Lemma 5.11, we obtain that $U^{(3)}$ has the center 1 . The statement follows by induction on $n \in \mathbb{N}$.

\subsection{INFINITE PALINDROMIC BRANCHES}

Every palindrome is either a central factor of a maximal palindrome $U^{(n)}$, or a central factor of an infinite palindromic branch. Their knowledge is essential for determination of the palindromic complexity of $u_{\beta}$.

Definition 5.14. Let $v=v_{1} v_{2} v_{3} \cdots$ be a right-sided infinite word over the alphabet $\{0,1\}$. Denote by $\bar{v}$ the left-sided infinite word $\bar{v}=\cdots v_{3} v_{2} v_{1}$. Let $z \in\{\varepsilon, 0,1\}$. If for every $n \in \mathbb{N}$, the palindrome $p=v_{n} v_{n-1} \ldots v_{1} z v_{1} v_{2} \ldots v_{n}$ belongs to $\mathcal{L}\left(u_{\beta}\right)$, then the bidirectional infinite word $\bar{v} z v$ is called an infinite palindromic branch of $u_{\beta}$ with the center $z$, and the palindrome $p$ is called the central factor of the infinite palindromic branch $\bar{v} z v$.

Lemma 5.15. Let $z \in\{\varepsilon, 0,1\}$. Then there exists at most one infinite palindromic branch with the center $z$.

Proof. It follows from Lemma 5.10 that applying the substitution $\varphi$ on an infinite palindromic branch, one obtains again an infinite palindromic branch. More precisely, from $\bar{v} z v$ one obtains $\overline{\varphi(v)} 10^{a} 1 \varphi(v)$ if $z=0, \overline{\varphi(v)} 10^{b} 1 \varphi(v)$ if $z=1$, and $\overline{\varphi(v)} 1 \varphi(v)$ if $z=\varepsilon$.

We show the statement of the lemma by contradiction. Suppose that there are two different infinite palindromic branches with the same center $z$. By applying the substitution $\varphi$, we obtain two different infinite palindromic branches with longer common central factor. Repeating this procedure, we can construct infinitely many different infinite palindromic branches, which is a contradiction with boundedness of the palindromic complexity.

We now describe the infinite palindromic branch of $u_{\beta}$ with the center $z \in\{\varepsilon, 0,1\}$ and the common central factors of this branch with maximal palindromes having the same center $z$. With this in hand, we will be able to summarize the values of the palindromic complexity. Note that the candidate for the longest common prefix of a maximal palindrome and an infinite palindromic branch with the same center is a palindrome, which has two palindromic extensions, thus one of the palindromes

$V^{(n)}$. Using Proposition 5.12, we can describe all infinite palindromic branches of $u_{\beta}$.

Proposition 5.16. 
(i) Let $b$ be even and $a$ odd. There exists an infinite palindromic branch with the center $z$ for all $z \in\{\varepsilon, 1,0\}$, namely the bidirectional limit:

$$
\begin{array}{ll}
\lim _{n \rightarrow \infty} V^{(2 n-1)} & \text { having the center } \varepsilon, \\
\lim _{n \rightarrow \infty} V^{(2 n)} & \text { having the center } 1, \\
\lim _{n \rightarrow \infty} W^{(n)} & \text { where } W^{(1)}=0, W^{(n)}=T\left(W^{(n-1)}\right), \\
& \text { having the center } 0 .
\end{array}
$$

For $n \in \mathbb{N}$, the longest common central factor of a maximal palindrome $U^{(n)}$ and the infinite palindromic branch with the same center is $V^{(n)}$. The limit of $W^{(n)}$ has a common central factor with no maximal palindrome.

(ii) Let both $b$ and $a$ be even. There exists an infinite palindromic branch with the center $z$ for $z \in\{\varepsilon, 1\}$, namely the bidirectional limit:

$$
\begin{array}{ll}
\lim _{n \rightarrow \infty} V^{(2 n-1)} & \text { having the center } \varepsilon, \\
\lim _{n \rightarrow \infty} V^{(2 n)} & \text { having the center } 1 .
\end{array}
$$

There is no infinite palindromic branch with the center 0 . For $n \in \mathbb{N}, n \geq 2$, the longest common central factor of a maximal palindrome $U^{(n)}$ and the infinite palindromic branch with the same center is $V^{(n-1)}$.

(iii) Let $b$ be odd and a even. There exists an infinite palindromic branch with the center $z$ for $z \in\{0, \varepsilon, 1\}$, namely the bidirectional limit:

$$
\begin{array}{ll}
\lim _{n \rightarrow \infty} V^{(3 n-2)} & \text { having the center } 0, \\
\lim _{n \rightarrow \infty} V^{(3 n-1)} & \text { having the center } \varepsilon, \\
\lim _{n \rightarrow \infty} V^{(3 n)} & \text { having the center } 1 .
\end{array}
$$

For $n \in \mathbb{N}$, the longest common central factor of a maximal palindrome $U^{(n)}$ and the infinite palindromic branch with the same center is $V^{(n)}$.

(iv) Let both $b$ and $a$ be odd. There exists an infinite palindromic branch with the center 0 , namely the bidirectional limit of palindromes $V^{(n)}, n \in \mathbb{N}$. There is neither an infinite palindromic branch with the center $\varepsilon$, nor with the center 1 . For $n \in \mathbb{N}, n \geq 3$, the longest common central factor of a maximal palindrome $U^{(n)}$ and the infinite palindromic branch is $V^{(n-2)}$.

Proof. It follows from Lemma 5.15 that there is at most one infinite palindromic branch with the center $z$ for each $z \in\{\varepsilon, 0,1\}$. In order to verify that the bidirectional limits of palindromes exist, it suffices to use Proposition 5.12 , or Lemma 5.10. to see that the palindromes are central factors of one another. Let us explain why in cases (ii) and (iv) one does not have an infinite palindromic branch with every center.

(ii) Let $b$ and $a$ be even, suppose that there is a palindromic branch with the center 0 . Necessarily, this branch has a block of the form $0^{a}$ or $0^{b}$ as its central factor. It is impossible owing to the fact that both $a$ and $b$ are even.

(iv) Let both $b$ and $a$ be odd, suppose that there is an infinite palindromic branch with the center $\varepsilon$. Then it has a block of the form $0^{a}$ or $0^{b}$ as its central factor. It is impossible since both $a$ and $b$ are odd. Suppose now that there exists an infinite palindromic branch with the center 1 . Take a central factor of this palindromic branch of the form $T(p)$ for a palindrome $p$ containing at least two letters 1 . Using Lemma [5.11] $p$ must have the center $\varepsilon$, and thus have a central factor $0^{a}$ or $0^{b}$, which is impossible.

The statements about the maximal common central factor of maximal palindromes and infinite palindromic branches are a consequence of Proposition 5.13 . 

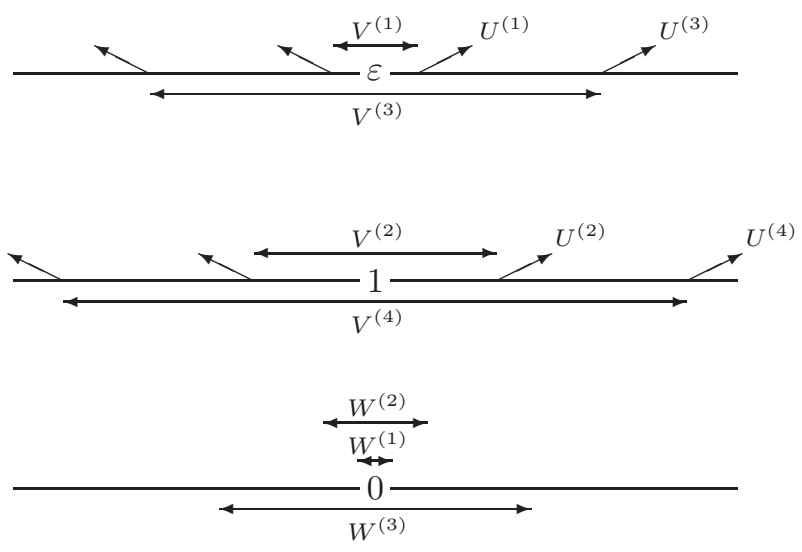

FIGURE 2. Illustration of maximal palindromes and infinite palindromic branches for $b$ even and $a$ odd. There is one infinite branch with center $\varepsilon$, one with center 1 , and one with center 0 . There are infinitely many maximal palindromes with center $\varepsilon$ and 1 .
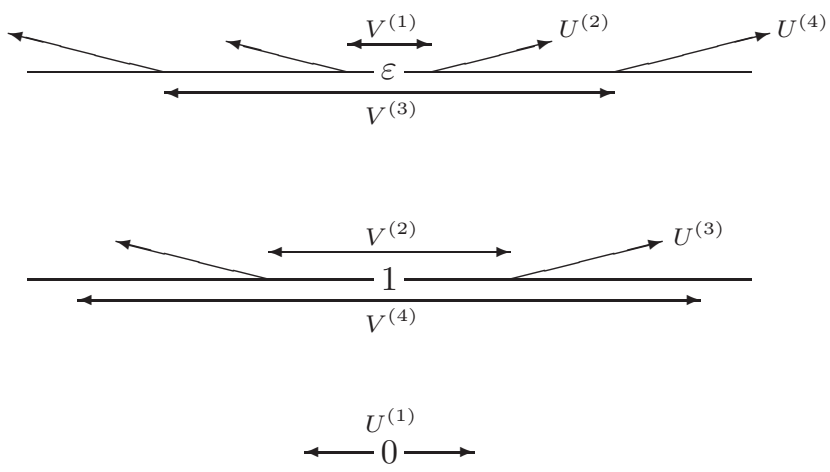

FIgURE 3. Illustration of maximal palindromes and infinite palindromic branches for $a$ and $b$ even. There is one infinite branch with center $\varepsilon$ and one with center 1 . There are infinitely many maximal palindromes with center $\varepsilon$ and 1 . There is only one maximal palindrome with center 0 .

\subsection{Explicit VAlues of the Palindromic COMPleXity of $u_{\beta}$}

We are now in position to derive explicitly the values of the palindromic complexity of the infinite word $u_{\beta}$ dependingly on the parity of parameters $a, b$ of the Rényi expansion of unity $d_{\beta}(1)=a b^{\omega}$. We have investigated maximal palindromes, infinite palindromic branches, and their centers. Determining the complexity is easy with the use of Figures 20 which visualize the structure of maximal palindromes and of infinite palindromic branches, according to Proposition 5.16 .

Theorem 5.17. Let $u_{\beta}$ be the fixed point of the substitution $\varphi(0)=0^{a} 1, \varphi(1)=$ $0^{b} 1, a-1>b \geq 1$. Then 4 cases can appear according to the values of parameters $a$ and $b, n \in \mathbb{N} \cup\{0\}$ : 

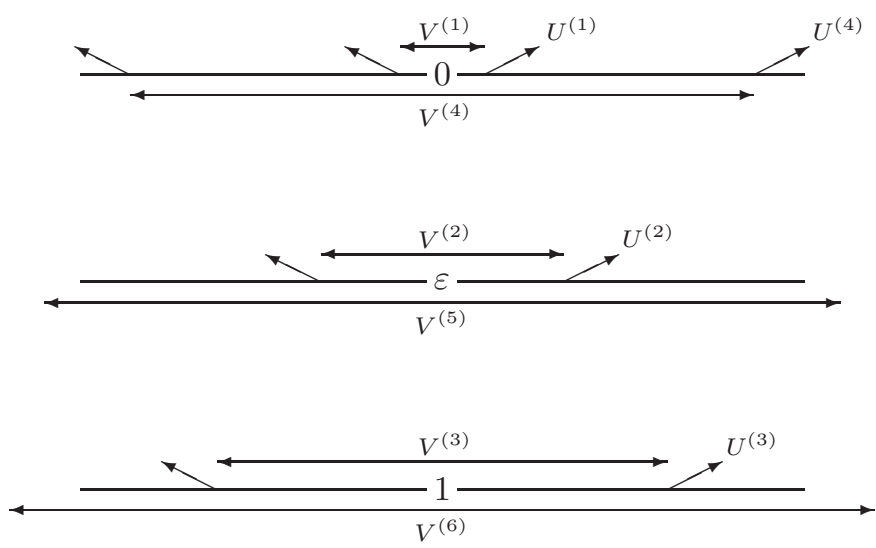

FiguRE 4. Illustration of maximal palindromes and infinite palindromic branches for $b$ odd and $a$ even. There are infinite branches and infinitely many maximal palindromes with centers $0,1, \varepsilon$.

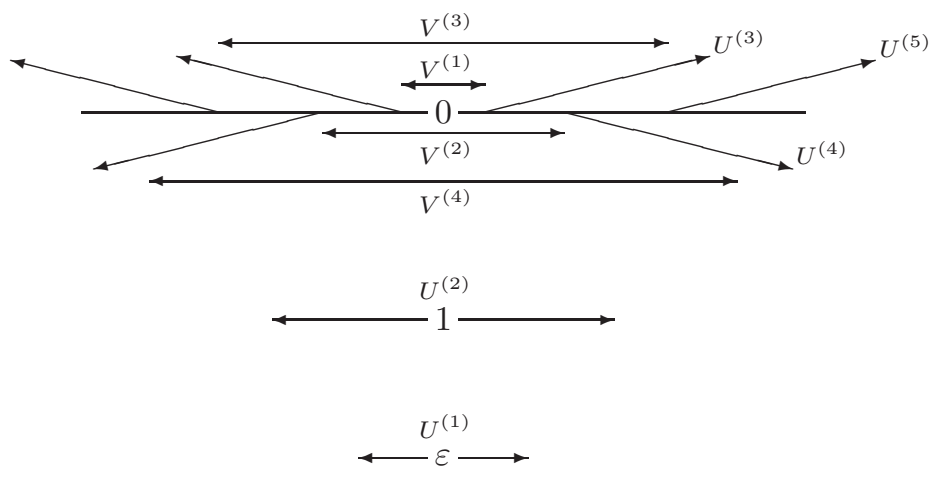

FiguRE 5. Illustration of maximal palindromes and infinite palindromic branches for $a$ and $b$ odd. The only infinite palindromic branch has center 0 . There are infinitely many maximal palindromes with center 0 . There is only one maximal palindrome with center $\varepsilon$ and one with center 1 .

(i) Let $b$ be even and a odd.

$$
\begin{aligned}
P(2 n) & = \begin{cases}2 & \text { if }\left|V^{(2 k-1)}\right|<2 n \leq\left|U^{(2 k-1)}\right| \text { for some } k \in \mathbb{N}, \\
1 & \text { otherwise. }\end{cases} \\
P(2 n+1) & = \begin{cases}3 & \text { if }\left|V^{(2 k)}\right|<2 n+1 \leq\left|U^{(2 k)}\right| \text { for some } k \in \mathbb{N}, \\
2 & \text { otherwise. }\end{cases}
\end{aligned}
$$

(ii) Let both $b$ and $a$ be even.

$$
\begin{aligned}
P(2 n) & = \begin{cases}2 & \text { if }\left|V^{(2 k-1)}\right|<2 n \leq\left|U^{(2 k)}\right| \text { for some } k \in \mathbb{N}, \\
1 & \text { otherwise. }\end{cases} \\
P(2 n+1) & = \begin{cases}2 & \text { if } 2 n+1 \leq\left|U^{(1)}\right|=a-1, \\
2 & \text { if }\left|V^{(2 k)}\right|<2 n+1 \leq\left|U^{(2 k+1)}\right| \text { for some } k \in \mathbb{N}, \\
1 & \text { otherwise. }\end{cases}
\end{aligned}
$$


(iii) Let $b$ be odd and a even.

$$
\begin{aligned}
P(2 n) & = \begin{cases}2 & \text { if }\left|V^{(3 k-1)}\right|<2 n \leq\left|U^{(3 k-1)}\right| \text { for some } k \in \mathbb{N}, \\
1 & \text { otherwise. }\end{cases} \\
P(2 n+1) & = \begin{cases}3 & \text { if }\left|V^{(k)}\right|<2 n+1 \leq\left|U^{(k)}\right| \text { for some } k \in \mathbb{N}, k \neq 2 \bmod 3, \\
2 & \text { otherwise. }\end{cases}
\end{aligned}
$$

(iv) Let both $b$ and $a$ be odd. We have

$$
\begin{aligned}
P(2 n) & = \begin{cases}1 & \text { if } 0 \leq 2 n \leq\left|U^{(1)}\right|=a-1, \\
0 & \text { otherwise. }\end{cases} \\
P(2 n+1) & = \begin{cases}2 & \text { if } 2 n+1 \leq\left|V^{(1)}\right|=b, \\
4 & \text { if }\left|V^{(k)}\right|<2 n+1 \leq\left|U^{(k)}\right| \text { for some } k \geq 2, \\
3 & \text { otherwise. }\end{cases}
\end{aligned}
$$

Note that we can either derive the values of the palindromic complexity for both even and odd $n$ directly from Proposition 5.16, or we can determine only $\mathcal{P}(2 n)$ and then use the relation (12) between palindromic complexity and the first difference of factor complexity,

$$
P(2 n+1)=\triangle C(2 n)+2-P(2 n)
$$

knowing the first difference of factor complexity from Theorem 4.3 .

\section{Conclusion}

The present paper completes the study of palindromic complexity of infinite words associated with $\beta$-integers for $\beta$ with eventually periodic Rényi expansion of unity in base $\beta$, i.e., $\beta$ a Parry number. The study started already in [1] for simple Parry numbers; here we focus on non-simple Parry numbers $\beta$. We focus on quadratic non-simple Parry numbers, since only in this case, one finds infinitely many palindromes in $u_{\beta}$. Such infinite words were studied for their balance properties in [4]. Other properties to be investigated are for example recurrent times or return words, which are sofar described only for a class of simple Parry numbers [5].

\section{ACKNOWLEDGEMENTS}

The authors acknowledge financial support by Czech Science Foundation GA ČR 201/05/0169, by the grant LC00602 of the Ministry of Education, Youth, and Sports of the Czech Republic.

\section{REFERENCES}

[1] P. Ambrož, Ch. Frougny, Z. Masáková, E. Pelantová, Palindromic complexity of infinite words associated with simple Parry numbers, to appear in Annales de l'Institut Fourier (2005), 25 pp.

[2] P. Baláži, Z. Masáková, E. Pelantová, Factor versus palindromic complexity of uniformly recurrent infinite words, to appear in Theoretical Computer Science (2006), 16 pp.

[3] L'. Balková, Complexity for infinite words associated with quadratic non-simple Parry numbers, submitted to Journal of Geometry and Symmetry in Physics (WGMP 2005 Proceedings) (2006), 12 pp.

[4] L. Balková, E. Pelantová, O. Turek, Combinatorial and arithmetical properties of infinite words associated with non-simple quadratic Parry numbers, to appear in RAIRO Theor. Inform. Appl. (2006) 
[5] L'. Balková, E. Pelantová, W. Steiner, Return words in the Thue-Morse and other sequences, submitted to Theor. Comp. Sci. (2006)

[6] J. Bernat, Étude sur le $\beta$-développement et applications, Mémoire de D.E.A., Université de la méditerrannée Aix-Marseille (2002)

[7] Č. Burdík, Ch. Frougny, J. P. Gazeau, R. Krejcar, Beta-integers as natural counting systems for quasicrystals, J. Phys. A 31 (1998), 6449-6472

[8] D. Damanik, L. Q. Zamboni, Combinatorial properties of Arnoux-Rauzy subshifts and applications to Schrődinger operators, Rev. Math. Phys. 15 (2003), 745-763

[9] D. Damanik, D. Zare, Palindrome complexity bounds for primitive substitution sequences, Discrete Math. 222 (2000), 259-267

[10] S. Fabre, Substitutions et $\beta$-systèmes de numération, Theoret. Comput. Sci. 137 (1995), 219-236

[11] Ch. Frougny, Z. Masáková, E. Pelantová, Complexity of infinite words associated with betaexpansions, RAIRO Theor. Inform. Appl. 38 (2004), 163-185; Corrigendum, RAIRO Theor. Inform. Appl. 38 (2004), 269-271

[12] Ch. Frougny, Z. Masáková, E. Pelantová, Infinite special branches in words associated with beta-expansions, to appear in J. Autom. Lang. Comb. (2005)

[13] A.Hof, O.Knill, B.Simon, Singular continuous spectrum for palindromic Schrödinger operators, Comm. Math. Phys. 174 (1995), 149-159

[14] J. Lagarias, Geometric models for quasicrystals I. Delone sets of finite type, Discrete Comput. Geom. 21 (1999), 161-191

[15] Y. Meyer, Quasicrystals, Diophantine approximation, and algebraic numbers, in: F. Axel, D. Gratias (Eds.), Beyond Quasicrystals, Les éditions de physique, Springer, Berlin (1995), 6-13

[16] W. Parry, On the beta-expansions of real numbers, Acta Math. Acad. Sci. Hungar. 11 (1960), 401-416

[17] A. Rényi, Representations for real numbers and their ergodic properties, Acta Math. Acad. Sci. Hungar. 8 (1957), 477-493

[18] K. Schmidt, On periodic expansions of Pisot numbers and Salem numbers, Bull. London Math. Soc. 12 (1980), 269-278

[19] D. Shechtman, I. Blech, D. Gratias, J. Cahn, Metallic phase with long-range orientational order and no translational symmetry, Phys. Rev. Lett. 53 (1984), 1951-1954

[20] W. P. Thurston, Groups, tilings, and finite state automata, Geometry supercomputer project research report GCG1, University of Minnesota (1989)

[21] O. Turek, Balance properties of the fixed point of the substitution associated to quadratic simple Pisot numbers, to be published in RAIRO Theor. Inf. Appl. (2006)

Communicated by (The editor will be set by the publisher).

(The dates will be set by the publisher). 